\title{
The gift of a lifetime: analysis of HIV at autopsy
}

\author{
Frank Maldarelli
}

HIV Dynamics and Replication Program, National Cancer Institute (NCI), NIH, Ft. Detrick, Maryland, USA.

\begin{abstract}
Currently, the incidence of HIV infection exceeds the death rate from HIV, and as a result, the prevalence of individuals living with the infection continues to increase. A critical limitation preventing the development of curative strategies is the lack of knowledge regarding mechanisms that allow HIV-infected cells to persist in individuals during combination antiviral therapy (ART). In this issue of the JCI, Chaillon and coworkers assessed HIV-infected cells from various anatomic compartments obtained through a rapid autopsy program of individuals undergoing long-term ART. This study, made possible with strong community collaboration, provides new insights on the potential locations of reservoirs of HIV-infected cells that persist during therapy.
\end{abstract}

\section{The Last Gift}

HIV currently infects over 37 million individuals worldwide (1). Although advances in the use of combination antiretroviral therapy (ART) have resulted in substantial improvements in morbidity and mortality from HIV infection, as the HIV epidemic has matured, new challenges have arisen. Adverse side effects from ART, the presence of ongoing immune activation contributing to comorbid disease, and the potential for the emergence of resistance to ART all contribute to decreased quality of life for infected individuals. As such, the current state of the epidemic remains unacceptable, and new approaches to cure HIV are a public health imperative.

There are now several examples of individuals who have been cured of HIV as the result of bone marrow transplantation for comorbid neoplasms, demonstrating that eradication is possible $(2,3)$. There are also examples of individuals who have controlled viremia without ART, either naturally $(4,5)$ or after a period of suppression of viremia with ART $(4,6)$. New studies to develop feasible and scalable strategies to cure or control HIV without ART represent fundamental research priorities. Strong commitments from governmental and private organizations support initiatives that are highly collaborative (e.g., AIDS Clinical Treatment Groups, Cure Collaboratories) and that directly engage communities of affected individuals have established a solid foundation to approach HIV eradication. The Last Gift autopsy program is one example of such a collaboration. In this issue of the JCI, Chaillon and colleagues explored HIV-infected cells in anatomic compartments obtained from six individuals who underwent a rapid autopsy procedure under the Last Gift protocol (7).

\section{Cycles of HIV infection}

HIV replication is rapid and error prone, and left untreated, infected cells produce genetically diverse viral populations that are widely distributed throughout the body. Although HIV infects cells of lymphoid and myeloid origin, highly activated lymphocytes comprise the vast majority of HIVinfected cells (>99.9\%) responsible for virus production. These cells are relatively

Related Article: p. 1699

Conflict of interest: The author has declared that no conflict of interest exists. 


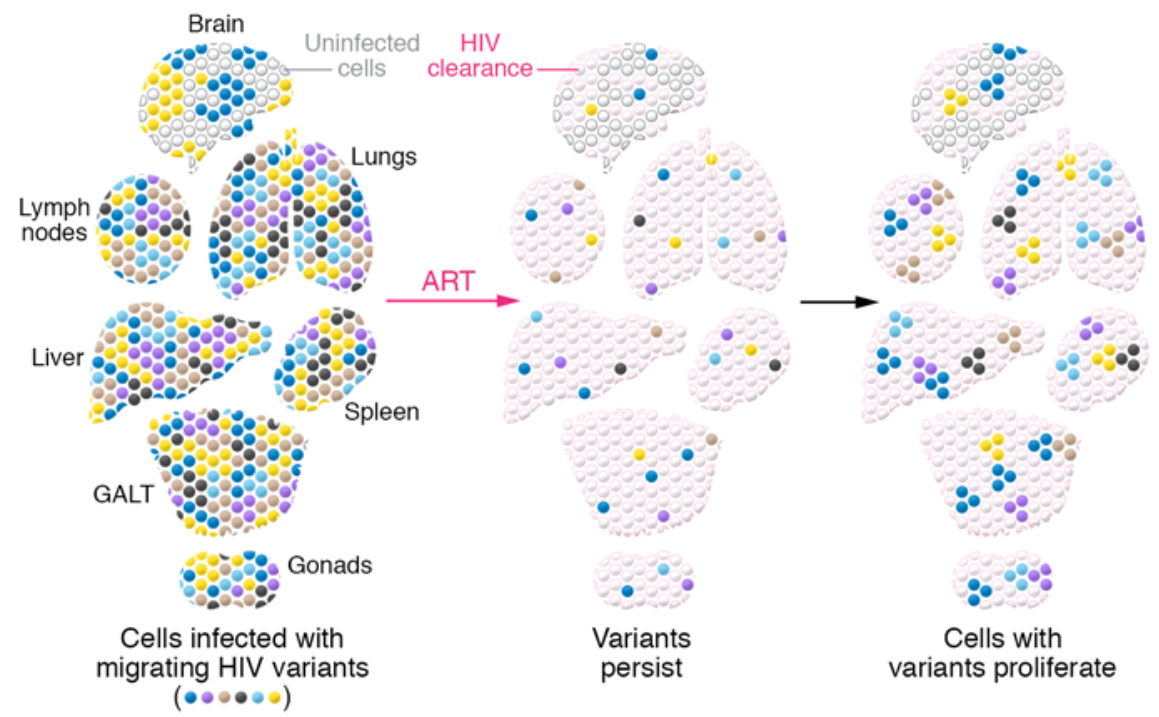

have failed to find evidence of active HIV replication cycles even within tissues (1822). Rather, cells infected by HIV prior to the introduction of ART persist during prolonged treatment and undergo clonal expansion (23-27). Clones arise early after HIV infection is established and persist and expand after ART is initiated (28). As a result, HIV is maintained during ART by cycles of cell replication, not cycles of virus replication.

Much of what has been learned regarding HIV cell populations, viral decay kinetics, persistence, and clonal expansion has been brought to light by analysis of plasma and peripheral blood lymphocytes, which contain only a small fraction (circa $2 \%)$ of infected cells. Information regarding the anatomic distribution of infected cells during prolonged ART is essential to understand the formation and maintenance of reservoirs of HIV-infected cells. Studies of tissues have identified HIVinfected cells, including clones, in lymphoid tissue as well as in pathologic tissue in individuals with comorbid neoplasms $(26,29)$, and putative clonal sequences could be detected in diverse tissues including lymphoid and CNS (21). Combined with longitudinal sampling of blood prior to expiration, such studies revealed that analysis of HIV-infected cells in tissues represents an archive of the history of HIV infection. In effect, autopsy-derived samples represent the "gift of a lifetime" of HIV infection in an individual; analysis of such tissues represents a compelling approach to investigate HIV persistence.

\section{Tracking HIV infection across tissues}

In this issue of the JCI, Chaillon et al. (7) obtained tissues from six individuals undergoing long-term ART as part of the Last Gift protocol, in which autopsies were performed within six hours of death. The study participants had significant comorbidities, and several chose to discontinue ART prior to death and had detectable viremia at the time of autopsy. Samples were obtained from 28 different tissues, including CNS tissue. The authors quantified HIV DNA in tissues and used endpoint dilution, single-genome sequencing to obtain a set (median of 7) of full-length HIV envelope DNA sequences from each tissue. Sequences were analyzed to determine phylogenetic and genetic relationships, and bioinformatic approaches were used to track how infected cells dispersed and migrated across tissues (7).

HIV DNA was detected in most tissues (7). Levels of HIV DNA in CNS tissue were low but detectable, consistent with previous findings that infection of the CNS occurs (30) and persists during therapy (31). All HIV DNA populations within individuals were highly related, but evidence of compartmentalization within tissues could be detected. In general, HIV DNA populations were genetically diverse, but Chaillon et al. also identified populations of proviruses in different tissues that were identical in sequence; in the setting of a genetically diverse population, repeated recovery of identical sequences suggests that these proviruses are members of a
Figure 1. Reservoirs of HIV-infected cells persist during ART. Cells infected with genetically diverse HIV variants including clones of cells are present throughout infected individuals even after prolonged ART. CALT, gut-associated lymphoid tissue. clone. These putative clonal members were detected in a number of tissues and provide useful insights into how infected cells are distributed in individuals (Figure 1) (7). Recently, Patro et al. (32) demonstrated that proviruses with nearly identical sequences may actually have different integration sites and may result from infection of identical viruses within different cells. Bioinformatic analyses are available to provide probabilities that identical sequences are clonal, and additional studies including integration site analysis will be necessary to identify authentic clones. In addition, correlations with primate studies, in which defined, "barcoded" viruses used for infection can permit precise tracking in anatomic locations (33), will shed additional insight on in vivo viral dissemination.

Notably, several of the study volunteers discontinued ART prior to death, and tissue analysis at autopsy revealed infected cells at multiple anatomical locations. Detailed bioinformatic analyses suggested that these locations can serve as hubs for viral dissemination as active replication cycles resume, and that these hubs are the source of rebound viremia after ART is discontinued (7). With single time-point analyses, it is often difficult to determine whether virus-infected cells in tissues are the origin or the destination of these newly infected cells; the patterns of dissemination and dispersal in tissues were complex in these study subjects. In addition, it is not clear what proportion of infected cells were producing HIV RNA. Previous stud- 
ies have demonstrated heterogeneity in the levels of RNA production, including in members of individual clones (34). Since the rapid autopsy strategy is likely to preserve HIV RNA, comparing HIV RNA from these tissues with stored specimens will permit detailed analyses of source and dispersal of HIV-infected cells and evaluation of new hypotheses. For instance, Abrahams and coworkers (35) performed longitudinal studies of HIV prior to and following ART introduction and proposed that the HIV variants induced from the latent reservoir after years of ART were most closely related to variants circulating at the time of ART initiation. Further characterization of HIV in the participants who discontinued therapy in the present study may yield useful information regarding the distribution of replication-competent HIV in tissues.

\section{Autopsy study limitations}

Autopsy studies pose new challenges and limitations. As described by J. Coffin, analysis of HIV in tissues is "solid-state virology" and, by its nature, carries concerns that are not encountered in studies of plasma or peripheral blood lymphocytes. Recovering single genomes of HIV from tissues as large as liver or as small as seminal vesicles is subject to potential sampling bias. Tissues taken at autopsy may contain varying amounts of residual blood, which itself may contain infected cells, thereby confounding tissue analysis. Additionally, the effect of comorbid diseases on the distribution of infected cells remains uncertain; for instance, one participant with pancreatic cancer in the present study showed the highest concentrations of HIV-infected cells in the pancreas (7). Future studies integrating solid-state virology with clinical data will make for a comprehensive analysis of these tissues.

As Chaillon et al. point out, a principal limitation of this and similar studies of disease pathogenesis is the overall paucity of individuals undergoing autopsy (7). For HIV research, this situation may be changing. The Last Gift program and other autopsy protocols provides an opportunity for individuals who may have been longstanding volunteers in other studies to offer an additional measure of participation; Last Gift enrollees have also described an additional sense of purpose (35). Similar sentiments have been voiced by participants in other HIV studies with autopsy options, such as those at the NIH Clinical Center. There are obvious and serious ethical concerns regarding recruitment for any autopsy protocol (36), but, in fact, investigators have learned that for HIV studies, the study participants themselves are often the first to raise the possibility of postmortem examinations. It is not surprising that the generation of HIV-infected individuals who have participated in research for many years would again lead the investigators, and be first to volunteer. As such, clinical investigators can consider discussions of autopsies as part of a routine evaluation in the appropriate study participants. On several levels, therefore, autopsies represent the gift of a lifetime.

\section{Acknowledgments}

We are grateful to the study participants and their families who make autopsy studies possible. We thank J. Coffin and S. Hughes for insightful discussions and the members of the Clinical Retrovirology Section of the HIV Dynamics and Replication Program, NCI, for helpful suggestions.

Address correspondence to: Frank Maldarelli, HIV Dynamics and Replication Program, NCI, NIH, 1050 Boyles St., Fort Detrick, Frederick, Maryland 21702, USA. Phone: 301.846.5611; Email:fmalli@mail.nih.gov.

1. [No authors listed]. WHO HIV/AIDS Statistics. World Health Organization. https://www.who. int/hiv/data/en/. Accessed February 11, 2020.

2. Gupta RK, et al. HIV-1 remission following CCR5 $\Delta 32 / \Delta 32$ haematopoietic stem-cell transplantation. Nature. 2019;568(7751):244-248.

3. Yukl SA, et al. Challenges in detecting HIV persistence during potentially curative interventions: a study of the Berlin patient. PLoS Pathog. 2013;9(5):e1003347.

4. May ME, Kwaa AK, Blankson JN. HIV-1 reservoirs in elite controllers: clues for developing a functional cure? Future Microbiol. 2017;12:1019-1022.

5. Migueles SA, Connors M. Success and failure of the cellular immune response against HIV-1. Nat Immunol. 2015;16(6):563-570.

6. Sáez-Cirión A, et al. Post-treatment HIV-1 controllers with a long-term virological remission after the interruption of early initiated antiretroviral therapy ANRS VISCONTI Study. PLoS Pathog. 2013;9(3):e1003211.

7. Chaillon A, et al. HIV persists throughout deep tissues with repopulation from multiple anatomical sources. J Clin Invest. 2020;130(4):1699-1712.

8. Sengupta S, Siliciano RF. Targeting the latent res- ervoir for HIV-1. Immunity. 2018;48(5):872-895.

9. Kruize Z, Kootstra NA. The role of macrophages in HIV-1 persistence and pathogenesis. Front Microbiol. 2019;10:2828.

10. Wallet C, et al. Microglial cells: the main HIV-1 reservoir in the brain. Front Cell Infect Microbiol. 2019;9:362.

11. Coffin JM. HIV population dynamics in vivo: implications for genetic variation, pathogenesis, and therapy. Science. 1995;267(5197):483-489.

12. Palmer S, et al. Low-level viremia persists for at least 7 years in patients on suppressive antiretroviral therapy. Proc Natl Acad Sci USA. 2008;105(10):3879-3884.

13. Riddler SA, et al. Continued slow decay of the residual plasma viremia level in HIV-1-infected adults receiving long-term antiretroviral therapy. J Infect Dis. 2016;213(4):556-560.

14. Anderson EM, et al. Dynamic shifts in the HIV proviral landscape during long term combination antiretroviral therapy: implications for persistence and control of HIV infections. Viruses. 2020;12(2):E136.

15. Besson GJ, et al. HIV-1 DNA decay dynamics in blood during more than a decade of suppressive antiretroviral therapy. Clin Infect Dis. 2014;59(9):1312-1321.

16. Bruner KM, et al. Defective proviruses rapidly accumulate during acute HIV-1 infection. Nat Med. 2016;22(9):1043-1049.

17. Imamichi H, et al. Defective HIV-1 proviruses produce novel protein-coding RNA species in HIV-infected patients on combination antiretroviral therapy. Proc Natl Acad Sci USA. 2016;113(31):8783-8788.

18. Henrich TJ, et al. HIV-1 persistence following extremely early initiation of antiretroviral therapy (ART) during acute HIV-1 infection: An observational study. PLoS Med. 2017;14(11):e1002417.

19. Kwon KJ, Siliciano RF. HIV persistence: clonal expansion of cells in the latent reservoir. J Clin Invest. 2017;127(7):2536-2538.

20. Wang Z, et al. Expanded cellular clones carrying replication-competent HIV-1 persist, wax, and wane. Proc Natl Acad Sci USA. 2018;115(11):E2575-E2584.

21. Bozzi G, et al. No evidence of ongoing HIV replication or compartmentalization in tissues during combination antiretroviral therapy: implications for HIV eradication. Sci Adv. 2019;5(9):eaav2045.

22. Kearney MF, et al. Ongoing HIV replication during ART reconsidered. Open Forum Infect Dis. 2017;4(3):ofx173.

23. Cohn LB, et al. HIV-1 integration landscape during latent and active infection. Cell. 2015;160(3):420-432.

24. Einkauf KB, et al. Intact HIV-1 proviruses accumulate at distinct chromosomal positions during prolonged antiretroviral therapy. J Clin Invest. 2019;129(3):988-998.

25. Maldarelli F, et al. HIV latency. Specific HIV integration sites are linked to clonal expansion and persistence of infected cells. Science. 2014;345(6193):179-183.

26. Simonetti FR, et al. Clonally expanded $\mathrm{CD} 4^{+} \mathrm{T}$ cells can produce infectious HIV-1 in vivo. Proc 
Natl Acad Sci USA. 2016;113(7):1883-1888.

27. Wagner TA, et al. HIV latency. Proliferation of cells with HIV integrated into cancer genes contributes to persistent infection. Science. 2014;345(6196):570-573.

28. Coffin JM, et al. Clones of infected cells arise early in HIV-infected individuals. JCI Insight. 2019;4(12):128432.

29. McManus WR, et al. HIV-1 in lymph nodes is maintained by cellular proliferation during antiretroviral therapy. J Clin Invest. 2019;130:4629-4642.

30. Wong JK, Ignacio CC, Torriani F, Havlir D, Fitch NJ, Richman DD. In vivo compartmentalization of human immunodeficiency virus: evidence from the examination of pol sequences from autopsy tissues. J Virol. 1997;71(3):2059-2071.

31. Joseph SB, et al. Human immunodeficiency virus type 1 RNA detected in the central nervous system (CNS) after years of suppressive antiretroviral therapy can originate from a replicating CNS reservoir or clonally expanded cells. Clin Infect Dis. 2019;69(8):1345-1352.

32. Patro SC, et al. Combined HIV-1 sequence and integration site analysis informs viral dynamics and allows reconstruction of replicating viral ancestors. Proc Natl Acad Sci USA. 2019;116(51):25891-25899.

33. Khanal S, et al. In vivo validation of the viral barcoding of simian immunodeficiency virus SIVmac239 and the development of new barcoded SIV and subtype B and C simian-human immunodeficiency viruses. J Virol. 2019;94(1):e01420-19.

34. Musick A, et al. HIV infected T cells can proliferate in vivo without inducing expression of the integrated provirus. Front Microbiol. 2019;10:2204.

35. Abrahams MR, et al. The replication-competent HIV-1 latent reservoir is primarily established near the time of therapy initiation. Sci Transl Med. 2019;11(513):eaaw5589.

36. Bach MC. Still human: a call for increased focus on ethical standards in cadaver research. HEC Forum. 2016;28(4):355-367. 